\title{
Etude des facteurs associés aux infections des plaies opératoires à l'Hôpital de Zone de Ouidah, Benin
}

\section{Study of factors associated with operating wound infections at the Ouidah Zone Hospital, Benin}

\author{
Haïdara DBS ${ }^{1}$, Agué $\mathrm{V}^{2}$, Touré L ${ }^{1}$, Mariko ML ${ }^{1}$, Cissouma $\mathrm{A}^{1}$
}

\section{DOI : 10.53318/msp.v11i1.1894}

1-Hôpital de Sikasso-Région de Sikasso -Mali

2-Institut Régional de Santé Publique (IRSP)-Ouidah - Université d'Abomey Calavi-Bénin

*Auteur correspondant : Dr Dadé Ben Sidi HAÏDARA dadhaidara@yahoo.fr ou haidaradade@gmail.com, Tel : (223)79 4174 07 ou (223) 66869390 ,

Direction Générale Hôpital de Sikasso BP : 22, tel : (223) 21620001 Ou (223)21 620117 ,

Fax: (223) 21620622

\section{RESUME}

INTRODUCTION : Les infections nosocomiales représentent un véritable problème de santé publique. L'objectif de cette étude était de déterminer les principaux facteurs de risque associés aux infections des plaies opératoires à l'hôpital zone de Ouidah de 2006 à 2007. MATERIEL et METHODES : II s'agissait d'une étude rétrospective, descriptive et analytique. Une régression logistique a été effectuée pour étudier les facteurs de risque de l'infection de la plaie opératoire. RESULTATS: Au total 521 dossiers ont été dépouillés entre 2006 à 2007. L'âge médian des opérés était de 26 ans avec un minimum de 1 an et un maximum de 90 ans. 54,7\% des opérés étaient hospitalisés dans le service de Gynécoobstétrique. Le sexe ratio était de 2 Femmes pour un Homme. La prévalence de l'infection a été $22,8 \%$. Le risque de survenue de l'infection de la plaie opératoire est plus élevé chez les opérés $>60$ ans (OR $=3,61$; IC à $95 \%$ $=[1,66-7,84])$. Ce risque est plus élevé $(\mathrm{OR}=10,78 ; \mathrm{IC}$ à $95 \%=[5,33-21,79])$ chez les opérés ayant séjourné plus d'une semaine en analyse multivariée. L'analyse qualitative montrait des facteurs dépendant à la fois des malades, du personnel et de l'organisation des services. CONCLUSION: La prévalence de l'infection de la plaie opératoire a été de $22,8 \%$. Les principaux facteurs de risques associés ont été : la durée de l'intervention chirurgicale, la durée d'hospitalisation, le rythme du pansement et la classe d'antibiotique utilisée en per opératoire.

MOTS CLES: Prévalence, Infection de la plaie opératoire, facteurs associés.

\footnotetext{
ABSTRACT

INTRODUCTION: Nosocomial infections represent a real public health problem. The objective of this study was to determine the main risk factors associated with infections of surgical wounds at the Ouidah zone hospital from 2006 to 2007. MATERIAL AND METHODS: This was a retrospective, descriptive and analytical study. Logistic regression was performed to study risk factors for surgical wound infection. RESULTS: A total of 521 files were
}

processed between 2006 and 2007. The median age of the operated was 26 years with a minimum of 1 year and a maximum of 90 years. $54.7 \%$ of those operated on were hospitalized in the Gyneco-obstetrics department. The sex ratio was 2 Women for a Man. The prevalence of infection was $22.8 \%$. The risk of developing surgical wound infection is higher in patients over 60 years of age (OR $=$ $3.61 ; 95 \% \mathrm{Cl}=[1.66-7.84])$. This risk is higher (OR = $10.78 ; 95 \% \mathrm{Cl}=[5.33-21.79])$ in those operated on who spent more than one week in multivariate analysis. The qualitative analysis showed factors depending on the patients, the staff and the organization of the services. CONCLUSION: The prevalence of surgical wound infection was $22.8 \%$. The main associated risk factors were: the duration of the surgical procedure, the length of hospitalization, the rate of the dressing and the class of antibiotic used during the operation.

KEY WORDS: Prevalence, Surgical wound infection, associated factors.

\section{INTRODUCTION}

Les infections nosocomiales représentent un véritable problème de santé publique avec des conséquences considérables tant sur le plan individuel que sur le plan économique. Les formations sanitaires sont conçues pour soigner et guérir les malades. Cependant, il est apparu depuis l'époque du Moyen-âge à nos jours qu'elles constituent des lieux ou le malade court un risque infectieux supplémentaire [1]. L'infection en Chirurgie a toujours constitué un problème pour le personnel de santé. Jusqu'au XIXème siècle, l'infection représentait dans les services chirurgicaux et obstétricaux, la première cause de décès [2].

Des études nationales de prévalence sur les infections nosocomiales ont été réalisées dans beaucoup de pays européens et en développement [3]. Elles ont servi à définir les priorités de la lutte contre les infections nosocomiales. La survenue des infections associées aux soins en milieu hospitalier est également favorisée par de nombreux facteurs dont les facteurs liés aux malades, les expositions aux dispositifs médicaux invasifs et les insuffisances dans la qualité des soins [4,5]. II en est de même des défauts de nettoyage, de désinfection et de stérilisation qui sont aussi des facteurs potentiels d'infection associée aux soins $[6,7,8]$. Les infections contractées en milieu hospitalier ont des conséquences variées, dont une augmentation de la morbidité et de la mortalité hospitalière, surtout dans les pays en voie de développement [9]. Ainsi, dans les hôpitaux de soins aigus, les infections associées aux soins sont la cinquième cause de décès [6]. 
Les sites anatomiques, à divers degrés, peuvent être intéressés par l'infection des plaies opératoires. De tous les sites anatomiques de développement des infections, le site chirurgical occupe le deuxième rang après l'appareil urinaire et avant les voies respiratoires inférieures. Les infections du site chirurgical continuent de représenter la principale complication des interventions chirurgicales et cela malgré les progrès scientifiques et technologiques enregistrés dans le domaine de la chirurgie [10].

Dans le Monde, en 2017 en France, un patient sur 20 hospitalisés en Etablissement de Santé (ES) était infecté $(4,98 \%$ IC95\% : $[4,62-5,36])$. Cette prévalence n'a pas diminué entre 2012 et 2017 alors que les enquêtes antérieures avaient montré une diminution constante depuis 2001 [11]

En Afrique, en République Démographique du Congo. Danny Kasongo Kakupa, a trouvé une prévalence globale de $34,5 \%$ en 2016 [12]. Au Bénin, la prévalence d'infections associées aux soins est de 14,39\% pour 139 patients inclus dans l'étude. Le sondage vésical en est un facteur de risque $(O R=2,45$; Chi-2=42,81 et $p<10-3)$. [13]. L'infection des plaies opératoires parait être donc un problème que doit gérer au quotidien les acteurs hospitaliers au Bénin. Par ailleurs II n'existe aucune donnée par rapport à ce type d'infection dans l'hôpital de zone de Ouidah. II apparait dès lors essentiel de déterminer la prévalence et les facteurs associés aux plaies opératoires dans ce type d'hôpital pour combler la lacune des connaissances par rapport à l'infection des plaies opératoires en vue de préconiser des mesures de prévention.

\section{MATERIEL ET MÉTHODES \\ Type et Cadre d'étude}

II s'agit d'une étude transversale rétrospective, descriptive et analytique sur deux ans, de 2006 à 2007. L'étude s'est déroulée dans l'hôpital de zone de Ouidah, situé au nordouest de la ville historique de Ouidah, elle-même située à une quarantaine de kilomètres de la capitale Cotonou. L'hôpital de zone de Ouidah comprend les infrastructures suivantes : un bloc administratif et un bloc médicotechnique comprenant un laboratoire, un service d'imagerie médicale, une pharmacie, un service de pédiatrie, de médecine, de chirurgie, de maternité, d'ophtalmologie, de stomatologie, de kinésithérapie, d'anesthésie réanimation et les unités annexes permettant le bon fonctionnement de l'hôpital. Le personnel est au nombre de 74 dont 6 médecins, 28 infirmiers 03 techniciens d'hygiène, 22 personnel d'appui et 12 administratifs.

\section{Population d'étude}

La cible primaire de l'étude était constituée par tous les patients ayant été opérés à l'hôpital Zone de Ouidah dans les services de Chirurgie et de Gynéco-obstétrique de 2006 à 2007.

Critères d'inclusion
Toutes les catégories de plaies opératoires sont incluses suivant la classification du Centre de Prévention et de Contrôle des maladies (CDC) [7]

\section{Critères d'infection}

Ecoulement purulent à partir d'une plaie chirurgicale ou d'un drain

et/ou

Ecoulement obtenu à partir d'une plaie ou d'un drain et dont la culture microbiologique est positive (plaie fermée seulement)

et/ou

Plaie nécessitant une réouverture

et/ou

Diagnostic d'infection par le chirurgien

\section{Critères de non inclusion}

Les plaies ne répondant pas aux critères d'infection du CDC. Les dossiers incomplets, n'ont pas pu être comptabilisés dans notre effectif de l'échantillon.

Période de l'étude : L'étude s'est déroulée d'octobre en novembre 2008

\section{Échantillonnage}

\section{Méthode d'échantillonnage}

La méthode d'échantillonnage a été non probabiliste avec deux techniques :

- Choix exhaustif des dossiers de tous les patients opérés à l'hôpital de Zone de Ouidah de 2006 à 2007.

- Choix raisonné de personnes ressources parmi les membres du personnel.

Taille de l'échantillon

$\mathrm{Au}$ total, 521 dossiers de patients opérés ont été dépouillés, 10 membres du personnel dans le service de chirurgie ont été interviewés. La cible secondaire est constituée par les membres du personnel de l'hôpital de Zone de Ouidah

\section{Collecte des données}

Trois techniques ont été utilisées : L'exploitation des documents pour les dossiers des malades et les registres des services de chirurgie et la maternité ; l'observation de l'environnement hospitalier et l'entretien avec le personnel de l'hôpital.

\section{Outils de collecte de données}

Conséquemment aux techniques, trois outils de collecte de données ont été utilisées : la Fiche de dépouillement ; la Grille d'observation et le Guide d'entretien.

\section{Déroulement de la collecte}

La collecte de données s'est déroulée du 13/10/2008 au $31 / 10 / 2008$

L'entretien avec les membres du personnel de l'hôpital a été réalisé le 03/11/2008 au 06/11/2008. L'observation de l'environnement hospitalier a couvert toute la période de collecte de données.

\section{Analyse des données}

Les proportions ont été calculées pour décrire les variables qualitatives et les moyennes suivies de l'écart type pour les variables quantitatives avec une distribution symétrique. Les variables quantitatives avec une distribution asymétrique ont été présentées avec leurs moyennes suivies des minimums et maximums. Pour 
l'analyse bi variée, nous avons utilisé les tests t de Student et le khi2 de KARL PEARSON pour les observations ayant une taille supérieure ou égale à 5 . Le test du khi carré corrigé de YATES a été utilisé pour les observations avec une taille inférieure à 5 . La prévalence de l'infection de la plaie opératoire exprimée en pourcentage a été estimée avec son intervalle de confiance à $95 \%$ (IC à $95 \%$ ). La mesure d'association donnée par la régression logistique est le rapport de cote. L'analyse statistique a été faite à l'aide du logiciel Epi info 2000 version 3.4.3.

Une régression logistique pas à pas descendante a été effectuée pour étudier les facteurs de risque de l'infection de la plaie opératoire. Les données des entretiens et de l'observation ont été analysées et triangulées de façon qualitative.

Les références bibliographiques sont présentées dans l'ordre d'appel selon la norme dite de Vancouver.

\section{Aspects éthiques}

Après explication des bénéfices de cette étude, on a eu le consentement éclairé de toutes les personnes interviewées et la confidentialité des données recueillies a été garantie en gardant l'anonymat des noms des malades.

\section{Conflit d'intérêt}

II n'y a aucun conflit d'intérêt avec les co-auteurs ou les institutions impliquées dans cette étude.

\section{RESULTATS}

Analyse descriptive des variables

Au total 521 dossiers des patients opérés de 2006 à 2007 ont été dépouillés soit 236 dossiers dans le service de Chirurgie et 285 dans celui de Gynéco-obstétrique.

Les caractéristiques sociodémographiques et les données cliniques des opérés

L'âge médian des opérés était de 26 ans avec un minimum de 1 an et un âge maximum de 90 ans. Parmi l'ensemble, $54,7 \%$ des opérés étaient hospitalisés dans le service de Gynéco-obstétrique. Plus de la moitié des opérés était de sexe féminin avec un sexe ratio de 2 .

La moitié des patients opérés venait directement des domiciles. Parmi les 521 opérés, 322 ont subi l'intervention chirurgicale de façon urgente. Sur l'ensemble des opérés, plus de la moitié ne souffraient d'aucune pathologie chronique associée à la maladie chirurgicale et aucun traitement spécifique n'était administré chez 366 opérés parmi les 521 . L'antibiothérapie était administrée dans $15,4 \%$ des cas.

(Tableau I)

\section{Les caractéristiques liées à l'infection}

Parmi les 521 opérés, 119 ont développé une infection de la plaie opératoire. Les structures anatomiques les plus concernées par l'infection étaient les organes et les cavités profondes dans $41,2 \%$ des cas infectés. On notait la présence de liquide /sérosité dans la plaie opératoire chez presque tous les opérés infectés. Le signe local d'infection le plus fréquemment évoqué par les opérés était la douleur au niveau de la plaie opératoire. Plus du quart des opérés infectés présentait une déhiscence de la plaie opératoire. Parmi les 119 opérés infectés, 19 ont subi une ré intervention chirurgicale de leurs plaies opératoires.

(Tableau II)

Les caractéristiques liées à l'acte chirurgical

La durée du séjour pré opératoire était de moins d'un jour chez 324 opérés parmi les 521. Le rasage du champ opératoire était effectué dans $86,6 \%$ des cas. Presque tous les opérés avaient reçu une antibiothérapie en per opératoire et les $\beta$ lactamines étaient les plus utilisées. Parmi les 521 opérés, 321 avaient une durée d'intervention de plus de deux heures. Le drainage chirurgical était appliqué chez 82 opérés parmi les 521et ce drain était retiré dans un délai inférieur ou égal à quatre jours chez 58 opérés parmi les 82 opérés dont les plaies opératoires avaient été drainées. La durée du séjour postopératoire était d'une semaine chez $45,1 \%$ des opérés. Tous les opérés avaient une durée de suivi postopératoire de 30 jours. Le pansement de la plaie était renouvelé chez 425 opérés parmi les 521 . La prothèse a été mise en place chez deux opérés soit $0,4 \%$. Presque tous les patients opérés sortaient guéris de l'hôpital soit 498. (Tableau III)

Répartition des types de plaies

Plus de la moitié des plaies chez les opérés était propre (Figure 1).

\section{Analyse bivariée}

Le risque de survenue de l'infection de la plaie opératoire est significativement plus élevé chez les opérés âgés de plus de 60 ans (OR $=3,61$; IC à $95 \%$ $=[1,66-7,84])$ que chez ceux âgés de 30 à 60 ans en prenant les opérés âgés de 1 à 30 ans comme référence.

- Le risque de survenue de l'infection de la plaie opératoire est significativement plus élevé $(O R=$ $1,58 ;$ IC à $95 \%=[0,41-0,96])$ chez les Femmes que chez les Hommes.

- Le risque de survenue de l'infection de la plaie opératoire est très significativement plus élevé $(\mathrm{OR}=$ $2,04 ;$ IC à $95 \%=[0,33-0,75])$ chez les opérés hospitalisés dans le service de Gynéco-obstétrique que ceux hospitalisés dans le service de Chirurgie. (Tableau IV)

- Le risque de survenue de l'infection de la plaie opératoire est très significativement plus élevé $(O R=$ $3,33$; IC à $95 \%=[0,18-0,50])$ chez les opérés n'ayant pas reçu un traitement antibiotique que ceux ayant reçu un traitement antibiotique.

- $\quad$ II n'y a pas de différence de risque pour la survenue de l'infection de la plaie opératoire entre les patients opérés en urgence et ceux opérés en programmation $(0 \mathrm{R}=0,93 ; \mathrm{IC}$ à $95 \%=[0,61-1,42])$.

- $\quad$ II n'y a pas de différence de risque pour la survenue de l'infection de la plaie opératoire entre les patients opérés avec une pathologie associée et ceux opérés sans pathologie associée $(0 \mathrm{R}=0,98$; IC à $95 \%=$ $[0,57-1,42])$.

- Le risque de survenue de l'infection de la plaie opératoire est très significativement plus élevé $(0 R=$ 
$1,95 ;$ IC à $95 \%=[1,28-2,99])$ chez les opérés ayant un traitement associé que ceux n'ayant pas un traitement associé. (Tableau V)

\section{Analyse multi variée}

Les variables explicatives retenues dans un premier temps sont : l'âge en 3 classes, le sexe, le service d'hospitalisation, le traitement associé en 2 classes, antibioprophylaxie, la classe d'antibiotique utilisée en 2 classes, le rasage du champ opératoire, le type de plaie en 2 classes, la durée d'hospitalisation en 2 clases, la durée de l'intervention en 2 classes, la mise en place de drain, et le rythme du pansement en 2 classes. Au cours de cette analyse, nous avons procédé à la recherche de la relation ajustée entre les facteurs et l'infection de la plaie opératoire. Elle a permis de retenir parmi tous les facteurs, ceux qui expliqueraient mieux la survenue de l'infection de la plaie opératoire (facteurs prédictifs).

Le modèle logistique complet est constitué par les variables dont le seuil de signification est de $20 \%$. Pour la sélection des variables non significatifs, nous avons utilisé le test de Vraisemblance. (Tableau VI)

La procédure de modélisation a été inspirée de l'approche d'Hosmer et Lemeshow permettant la sélection du meilleur modèle en se basant sur le maximum de vraisemblance. A partir du modèle complet, nous avons procédé à la détermination des $p$ values réels pour les variables ayant plus de deux modalités dans le modèle avec les résultats des Chi2 de Wald. Ensuite, nous avons sélectionné par la méthode dégressive pas à pas pour éliminer les variables dont le $p$ value n'est pas significatif ( $p$ value $>0,05$ ). La recherche des facteurs de confusion et de modificateurs d'effet a été effectuée à partir de l'appréciation de la variation relative (VR) de l'apport de chaque variable à enlever dans le modèle. Nous avons retenu un seuil de $20 \%$ pour la VR de chaque variable. Les variables retenues dans le modèle final sont présentées dans le (Tableau VII).

La variable antibioprophylaxie a été retenue dans ce modèle final car elle constitue un facteur de confusion pour la variable durée de l'intervention. La variable rasage du champ opératoire est un facteur de confusion pour la variable rythme de pansement, raison pour laquelle nous l'avons maintenu dans ce modèle.

Ajusté sur les autres facteurs, II n'y a pas de différence de risque $\left(\mathrm{OR}_{\mathrm{a}}=0,00 ; \mathrm{IC}\right.$ à $95 \%=[0,00$ - 1,0]) de survenue de l'infection de la plaie opératoire entre les opérés ayant subi une antibioprophylaxie et ceux n'ayant pas subi l'antibioprophylaxie.

- Le risque de survenue de l'infection de la plaie opératoire est significativement plus élevé $\left(0 \mathrm{R}_{a}=\right.$ $1,92 ;$ IC à $95 \%=[1,06-3,49])$ chez les opérés ayant un traitement avec les autres classes d'antibiotique comparativement à ceux ayant reçu un traitement avec les $\beta$-lactamines en ajustant sur les autres facteurs.

- Le risque de survenue de l'infection de la plaie opératoire est très significativement plus élevé (ORa
= 10,78; IC à 95\% = [5,33 - 21,79]) chez les opérés ayant séjourné plus d'une semaine comparativement à ceux ayant séjourné une semaine à l'Hôpital en ajustant sur les autres facteurs dans le modèle.

- Le risque de survenue de l'infection de la plaie opératoire est très significativement plus élevé (ORa $=6,80 ;$ IC à $95 \%=[1,43-32,46])$ chez les opérés ayant une durée d'intervention de plus de deux heures que ceux ayant une durée d'intervention de plus d'une heure en ajustant sur les autres facteurs. Le risque de survenue de l'infection de la plaie opératoire est très significativement plus élevé ( $\mathrm{ORa}$ $=8,33$; IC à $95 \%=[0,06-0,24]$ ) chez les opérés ayant un rythme de pansement d'un jour sur quatre que ceux ayant un rythme de pansement d'un jour sur deux en ajustant sur les autres facteurs : risque protecteur de 0,12 équivalent à un facteur de risque de 8,33 soit $(1 / 0,12)$.

- $\quad$ Ajusté sur les autres facteurs, le risque de survenue de l'infection de la plaie opératoire est plus élevé $\left(\mathrm{OR}_{\mathrm{a}}=1,86 ; \mathrm{IC}\right.$ à $\left.95 \%=[0,78-4,41]\right)$ chez les opérés ayant subi un rasage du champ opératoire par rapport à ceux n'ayant pas subi un rasage du champ opératoire mais la différence n'est pas statistiquement significative.

Mesure du niveau de connaissance du personnel sur l'hygiène hospitalière

Les facteurs et les situations incriminées dans la survenue des infections des plaies opératoires dépendent à la fois des malades, du personnel et de l'organisation des services :

Parmi les facteurs organisationnels nous avons retenu :

- Les références tardives depuis les formations sanitaires périphériques ;

- L'insuffisance des boites de pansements ;

- L'insuffisance et l'inadaptation des produits utilisés pour la décontamination ;

- Le nombre relativement élevé de malades dans les salles d'hospitalisation.

\section{DISCUSSION}

Des progrès sensibles ont été accomplis dans l'accroissement de l'espérance de vie et la réduction de certaines causes majeures de la mortalité infantile et maternelle, ainsi que d'autres maladies [13]. L'apparition de la pénicilline en 1945 a montré que les grandes infections semblaient vaincues, mais parallèlement avec le développement des techniques invasives et l'insuffisance des mesures d'hygiène hospitalière dans certains cas, les infections associées aux soins sont redevenues un problème de santé publique. Cette situation accentuée avec l'émergence des bactéries pathogènes résistantes aux antibiotiques, oblige l'équipe soignante à une attitude thérapeutique plus rigoureuse dans la prise en charge des patients [13]. L'objectif de l'étude a été de déterminer la prévalence et les facteurs associés à ces infections des plaies operatoires à l'hôpital 
de zone de Ouidah. La prevalence de infection a été de $22,8 \%$.

\section{LA PREVALENCE DE L'INFECTION DE LA PLAIE OPERATOIRE :}

En Afrique, la prévalence des infections associées aux soins varie entre $2,5 \%$ et $14,8 \%$ [14].

Au Bénin, la prévalence enregistrée chez les patients hospitalisés au Centre National Hospitalier et Universitaire Hubert Koutoukou Maga (CNHU-HKM) en 2012 était de 9,84\% [15].

Au plan national, dans $87 \%$ des hôpitaux inclus dans une enquête, elle était de 19,1\% [16].

Cette proportion est supérieure au $5,7 \%$ obtenue dans une étude réalisée en 2007 au CHU Hassan II de Fès au Maroc [3]. Cette différence pourrait être due à l'aspect prospectif de son étude et tous les facteurs incriminés étaient explorés. BIRINTANYA N. en 2002 a rapporté une prévalence d'infection de la plaie opératoire de 10,1\% au Centre Hospitalier Départemental de L'OUEME du PLATEAU au Bénin [18]. Celle-ci est moins importante que la prévalence de notre étude.

En Tunisie, HOUET K. et all ont rapporté une prévalence de l'infection des plaies opératoires en chirurgie digestive de 3,53 \% [24] qui est largement en deçà de notre taux. Notre prévalence, est largement en deçà de rapporté par d'autres études : En Suisse, une étude de prévalence des infections nosocomiales réalisée en 1996 dans 4 hôpitaux universitaires a montré que les infections du site opératoire figuraient en première place, représentant $30 \%$ de toutes les infections [21]. Une étude réalisée au Centre Hospitalier Universitaire (CHU) Bab El Oued d'Alger a montré que les infections de plaies opératoires étaient les plus fréquentes, constituant $35,4 \%$ de l'ensemble des infections [19]. L'estimation de la prévalence de l'infection de la plaie opératoire dans notre échantillon est plus élevée que celle trouvée dans d'autres études : En 1999 et au même Centre Hospitalier, N'DA M'PO I. nota une incidence de 10,52 \% [2]. Au Burkina Faso, DAO B. et all ont rapporté une prévalence de 11,25\% d'infection des plaies opératoires [25]. MUTOMBO DP. Et all au Congo Démocratique (ex Zaïre) ont trouvé une prévalence de 19 $\%$ [26]. Les réseaux de surveillance de l'incidence de l'infection nosocomiale en France montrent qu'entre 1999 et 2002, chez les patients à faible risque d'infections nosocomiales, le taux d'incidence des infections du site opératoire (ISO) est de 0,66 pour 100 interventions (sur une base de 126144 interventions) et varie selon l'intervention : pour les prothèses de hanche, le taux d'ISO est de $0,51 \%$ et pour les césariennes de $1,83 \%$ [27]. Le Comité d'Infectiologie de l'Association Française d'Urologie (CIAFU) a rapporté une prévalence de malades infectés en Chirurgie de 6,1\% [28]. D'autres études ont trouvé un taux supérieur à celui de notre étude : OTHEPA $\mathrm{M} O$. et RUI T. ont estimé qu'environ $38,6 \%$ des plaies opérés étaient infectées [29]. Le Réseau d'Alerte, d'investigation et de surveillance des Infections Nosocomiales (RAISIN) a estimé que parmi l'ensemble des IN identifiées chez les opérés les ISO représentaient $25,4 \%$ [30].

\section{LES FACTEURS DE RISQUE LIES A L'ACTE CHIRURGICAL}

Le rasage préopératoire influence également le risque opératoire. Plusieurs études ont montré une augmentation du risque lorsque les poils étaient enlevés avec un rasoir à main par rapport à un rasoir électrique ou une épilation. De plus, lorsqu'un rasoir à main était utilisé, le risque était double lorsque le rasage était effectué dans les 24 heures qui précédaient l'opération par rapport à immédiatement avant l'opération.

Dans notre série, le risque de survenue de l'infection de la plaie opératoire est très significativement élevé $(O R=$ $1,82 ;$ IC à $95 \%=[0,32-0,96])$ chez les opérés ayant subi un rasage du champ opératoire par rapport à ceux n'ayant pas subi un rasage du champ opératoire. Ceci est conforme avec les résultats apportés par d'autres études $[10,29]$.

Le risque de survenue de l'infection de la plaie opératoire est très significativement plus élevé $(\mathrm{OR}=3,06$; IC à $95 \%$ $=[2,01-4,67])$ chez les opérés ayant une plaie sale/infectée comparativement à ceux ayant une plaie propre. Ceci apporte une conformité avec la littérature $[2,10,18,31]$. L'infection de la plaie opératoire rallonge la durée du séjour postopératoire. Plus le séjour postopératoire est long, plus le risque de survenue de l'infection de la plaie est élevé. C'est ainsi que dans notre enquête, nous avons trouvé que le risque de survenue de l'infection de la plaie opératoire est très significativement plus élevé $(O R=9,16$; IC à $95 \%=[5,07-16,54])$ chez les opérés ayant séjourné plus d'une semaine comparativement à ceux ayant séjourné une semaine à l'Hôpital. Plusieurs auteurs avaient mentionné dans leurs études [2,10,18,26,31].

Le risque d'infection de la plaie opératoire semble particulièrement accru pour les opérations qui durent plus de deux heures. Dans cette étude impliquant 521 opérations, le risque lié à une opération de plus de deux heures est de 6,39 (IC à 95\% = [1,96 - 20,79]). Plusieurs facteurs sont évoqués pour expliquer cette augmentation du risque : augmentation de la contamination de la plaie, augmentation du traumatisme chirurgical, augmentation du nombre de sutures et de procédures d'électrocoagulation, augmentation des pertes sanguines, diminution de l'effet des antibiotiques prophylactiques. Ce résultat concorde avec celui rapporté par d'autres études $[2,3,10,18,26,31]$.

Nous n'avions pas trouvé de différence de risque entre les patients opérés en programmation et ceux opérés en urgence par rapport à la survenue de l'infection de la plaie opératoire au décours de notre enquête $(p=0,74)$. Cela pourrait probablement s'expliquer par la disponibilité d'un kit d'urgence remboursable au niveau du bloc opératoire. Ce qui permettra à tous les patients opérés d'accéder aux premiers soins au bloc. Une antibioprophylaxie chirurgicale efficace doit permettre de réduire de façon significative le risque d'infection de la plaie opératoire. 
C'est un point de vue unanimement partagé par les auteurs $[10,18,24,31,32]$. Notre étude a que le risque de survenue de l'infection de la plaie opératoire est très significativement plus élevé : 7,14 (IC à $95 \%=[0,04-$ $0,47]$ ) chez les opérés n'ayant pas reçu une antibioprophylaxie en per opératoire. II en de même pour les classes d'antibiotique utilisées. Ce risque a été de 2,52 (IC à 95\% = [1,52-3,91]) pour les opérés traités avec les autres familles d'antibiotiques comparés à ceux mis sous les $\beta$-lactamines. Le risque lié à la mise en place d'un drain a été de 8,98 chez les opérés ayant reçu un drain comparativement à ceux n'ayant pas été drainé (IC à $95 \%$ $=[5,36-15,03])$. Dans la littérature, des études ont rapporté le même résultat $[10,26]$.

Les opérés ayant un rythme de pansement d'un jour sur quatre (pansement au 5 ème jour) ont un risque de 8,33 (IC à $95 \%=[0,08-0,20])$ d'avoir leurs plaies infectées par rapport à ceux ayant un pansement d'un jour sur deux (pansement 48 ème Heure). FANCIOLI $P$. et al [10] avaient tiré la même conclusion. Un séjour préopératoire prolongé augmente le risque d'infection en dehors de la présence d'autre facteur de risque. Dans notre série, le risque de survenue de l'infection de la plaie opératoire est plus élevé $(O R=1,46 ;$ IC à $95 \%=[0,83-2,59])$ chez les opérés ayant séjourné plus d'un jour à l'hôpital avant l'opération comparés à ceux ayant séjourné un jour avant l'acte chirurgical, en prenant comme référence les opérés séjournant moins d'un jour mais la différence n'est pas significative. Cependant dans la littérature d'autres auteurs ont trouvé ce lien significatif $[2,10,18]$. Cette différence pourrait s'expliquer par la difficulté liée à la détermination de la date d'admission avant l'acte opératoire dans notre étude.

\section{MODELE PREDICTIF DE L'INFECTION DE LA PLAIE OPERATOIRE}

L'analyse multi variée nous a permis d'établir un modèle prédictif de l'infection de la plaie opératoire. Les facteurs prédictifs retenus sont : la durée de l'intervention $(O R=$ $6,80 ;$ IC à $95 \%=[1,43-32,46])$, la durée d'hospitalisation $(\mathrm{OR}=10,78 ; \mathrm{IC}$ à $95 \%=[5,33-21,79])$, le rythme du pansement $(O R=0,12 ; \mathrm{IC}$ à $95 \%=[0,06-0,27])$, la classe d'antibiotique utilisée en per opératoire $(\mathrm{OR}=1,92$; IC à $95 \%=1,06-3,49]$ ). Les facteurs antibioprophylaxie et le rasage du champ opératoire, ajustés sur les autres facteurs n'ont pas été significatifs.

Ils constituent des facteurs de confusion respectivement pour la durée de l'intervention et le rythme du pansement. La majorité des facteurs que nous avons identifié sont clairement retrouvés comme facteurs de risque dans la littérature tels que la durée de l'intervention $[2,3,10,18$, $26,31]$, la durée d'hospitalisation $[2,10,18,26,31]$, le rythme du pansement [10]. La classe d'antibiotique utilisée est un facteur prédictif mais peu abordé dans la littérature à cause de multiples biais de confusion. L'antibioprophylaxie est un facteur bien retenu par plusieurs études [10, 18, 24, 31, 32] mais à l'issue de notre modélisation, ajustée sur les autres facteurs, elle n'a pas été significative. Cela pourrait probablement être dû au nombre élevé de patients opérés mis systématiquement sous antibiotiques en per opératoire. Donc liée à l'organisation des soins à l'hôpital où tous les opérés ont accès à un kit d'urgence.

Un échantillon plus important que celui de notre enquête pourrait minimiser autant que possible les biais de sélection et d'information relatifs à notre étude.

Données qualitatives : Le personnel a une connaissance sur le risque infectieux hospitalier et il existe un comité de lutte contre ces infections mais la prise de conscience n'est pas effective. FRANCIOLI P. et al [10] avaient suggéré que le lavage des mains entre deux pansements et le port de gants stériles réduiraient significativement la fréquence des infections nosocomiales.

Les études de prévalence restent, malgré leurs limites, le moyen le plus simple à choisir dans le cadre de cette surveillance pour déterminer l'ampleur des infections nosocomiales spécifiquement celles des plaies opératoires lorsque les ressources sont réduites.

\section{Limites de l'étude}

Notre étude étant rétrospective, elle n'est pas à l'abri des biais concernant l'auto-sélection des populations témoins et le recueil de l'information « exposition au facteur » qui est différente entre les malades et les témoins.

L'échantillonnage étant exhaustif, tous les dossiers des opérés devraient être inclus.

\section{CONCLUSION}

Parmi les 521 opérés, 119 ont développé une infection de la plaie opératoire $(22,8 \%)$.

Ainsi, nous avons identifié les principaux facteurs de risques associés dont la durée de l'intervention chirurgicale, la durée d'hospitalisation. Les mesures d'hygiène et d'asepsie ont été insuffisantes ce qui augmenterait le risque de survenue de l'infection de la plaie opératoire.

\section{CONTRIBUTIONS DES AUTEURS}

Pr Agué VICTOIRE-Institut Régional de Santé Publique (IRSP) -Ouidah-Université d'Abomey Calavi-Bénin, co investigatrice,

Dr Dadé Ben Sidy Haidara, écriture du manuscrit

Dr Layes Touré, Hôpital de Sikasso-Mali, relecture et correction,

Dr Mohamed Lamine Mariko, Hôpital de Sikasso-Mali, relecture et correction,

Dr Assetou Cissouma, Hôpital de Sikasso-Mali, relecture et correction.

\section{REMERCIEMENTS}

Toute l'équipe du Master de Santé Publique de Ouidah, les personnels de l'IRSP, de l'Hôpital de Zone de Ouidah et de l'Hôpital de Sikasso. 


\section{REFERENCES}

1. TORLOTIN J-C. Hospitalisation et risque infectieux. In: DAUPHIN A., DARBORD J-C. Hygiène pratique. 2é ed. Paris : Editions Médicales internationals, 1998 : 1-28

2. N'DAM'PO I. Etude du coût des infections Postopératoires des plaies au Centre National Hospitalier et Universitaire (CNHU) de Cotonou. [Mémoire de Maîtrise en Sante Publique]. Cotonou : IRSP N $N^{\circ} 195$ /UAC, $1999: 54 p$

3. El Rhazi K., Elfakir S., Berraho M., Tachfouti N., Serhier Z., Kanjaa C. et al.

Prévalence et facteurs de risque des infections nosocomiales au CHU Hassan II de Fès (Maroc) La Revue de Santé de la Méditerranée orientale 2007 ; 13(1) : 56-63 4. Durocher A, 2005. L'infection nosocomiale comme indicateur de (non) qualité des soins : l'exemple de la réanimation (Commentaire). In: Sciences sociales et santé. Volume 23(3) : 59-68.

5. Espinasse F., Page B., Cottard-Boulle B, 2010. Risques infectieux associés aux dispositifs médicaux invasifs Revue francophone des laboratoires 426 : 51-63. 6. Sydnor ER, et Perl TM 2011. Hospital Epidemiology and Infection Control in acute- care Settings. Clin Microbiol Rev. 24(1) : 141-73.

7. Ghazi M., Khanbabaee G., Fallah F., Kazemi B., Mahmoudi S., Navidnia M., et al 2012. Emergence of Pseudomonas aeruginosa cross-infection in children with cystic fibrosis attending an Iranian referral pediatric center. Iran J Microbiol. 4(3) : 124-129.

8. Abreu AC., Tavares RR., Borges A., Mergulhão F., Simões M. 2013. Current and emergent strategies for disinfection of hospital environments. J Antimicrob Chemother. 68(12) :2718-32

9. Hamza R, 2010. Epidémiologie des infections associées aux soins. Revue Tunisienne d'Infectiologie. 4 : $1-4$.

10. Francioli P., Nahimana I., Lausanne, Widmer A., Bâle.

Infections nosocomiales et hygiène hospitalières : aspects actuels

Infections du site chirurgical : revue Mars 1996 ; 3(1)

11. Côme Daniau., Lucie Léon., Hervé Blanchard., Claude Bernet., Emmanuelle Caillat-Vallet., Sophie Glorion. et al.

Infections associées aux soins en établissement de santé : résultats de l'enquête nationale de prévalence 2017, France

12. Danny Kasongo Kakupa., Prosper Kalenga Muenze.,Baudouin Byl., et Michèle Dramaix Wilmet Etude de la prévalence des infections nosocomiales et des facteurs associes dans les deux hopitaux universitaires de Lubumbashi, République Démocratique du Congo: cas des Cliniques Universitaires de Lubumbashi et l'Hôpital Janson Sendwe

13. F Cyr Doscoph Afle., Kisito J.M.K. Quenum., Septime Hessou., Roch Christian Johnson État des lieux des infections associées aux soins dans deux hôpitaux publics du sud Benin (Afrique de l'ouest):
Centre Hospitalier Universitaire de Zone d'AbomeyCalavi/Sô-Ava et Centre Hospitalier de Zone de Cotonou 5

14. Bagheri Nejad S., Allegranzi B., Syed SB, Ellis B., Pittet D., 2011. Health-care-associated infection in Africa : a systematic review. Bull World Health Organ. 89(10) : 757-65.

15. Ouendo EM., Saïzonou J., Dégbey C., Glélé Kakai C., Glélé Y., MAKOUTODE M., 2015. Gestion du risque infectieux associé aux soins et services au Centre National Hospitalier et Universitaire Hubert Koutoukou Maga de Cotonou (Bénin), Int. J. Biol. Chem. Sci. 9(1) : 292-300.

16. Ahoyo Th A., Bankolé H S., Adéoti F M., Gbohoun A A., Assavèdo S., Amoussou-Guénou., et al 2014. Prevalence of nosocomial infections and anti-infective therapy in

Benin: results of the first nationwide survey in 2012 Antimicrobial Resistance and Infection Control $3: 17$. 17. Ministère de la santé, de la jeunesse et des sports de la république Française. Infections nosocomiales : le dossier Janvier-2008:1-37

18. BIRINTANYA N. Etude de la prévalence des infections des plaies postopératoires au Centre Hospitalier Départemental de l'Ouémé et Plateau [Mémoire de Maitrise en Santé Publique]. Cotonou : IRSP N ${ }^{\circ} 270$ / UAC, 2002

19. EZZAOUCHA A., MAKHLOUF F., DEKKAR N., LAMDJADANI N.

Prévalence des infections nosocomiales au centre hospitalo-universitaire de Bab El Oued-Alger La revue de la Médecine et maladies infectieuses 1994 ; 24(2) : 96-101 20. Université Claude Bernard Faculté de Médecine Lyon Sud

SANTE PUBLIQUE : Epidémiologie Economie de la santé Médecine préventive

Les infections nosocomiales et la gestion de la qualité et des risques: AMEUSO 2007.

21. BARBUT F., CARBONNE B., TRUCHOT F., SPIELVOGEL C., JANNET D., GODEREL I. et all Infections de site opératoire chez les patientes césarisées : bilan de 5 années de surveillance

Journal de gynécologie obstétrique et biologie de la reproduction $2004 ; 33(6)$ : 487-496

22. LEMARIE C., TEGUETE I., CHAMPION 0., FOURNIE A., DESCAMPS Ph., SIX $P$.

Infections du site opératoire après césarienne : intérêt d'une surveillance après la sortie de l'hôpital. Hygiènes 2006 ; 14(4): 253-256

23. DIA N M., KAB R., DIENG C., DIAGNE R., DIA M L., FORTES L. et al.

Résultats de l'enquête de prévalence des infections nosocomiales au CHNU de Fann (Dakar, Sénégal) Prévalence of nosocomial infections in university hospital (Dakar, Sénégal): Médecine et maladies infectieuses 2008: 38: 270-274

24. HOUET K., KACEM C., KOLSI K., HASSAIRI M., AYADI M M., EL HACHAICHI A. et al Facteurs de risque 
de l'infection des plaies opératoires en chirurgie digestive : étude rétrospective sur 3000 plaies opératoires.

Tunisie médicale $2000 ; 78$ (11):634 - 640

25. DAO B., TOURE B., BAMBARA M., YARA JP., KOALAGA AP., BAZIE AJ.

Indication et suites opératoires de la césarienne en milieu Africain : l'expérience de la maternité du Centre Hospitalier National SOURO SANOU de Bobo-Dioulasso au Burkina Faso Louvain médical 1998 ; $117: 96$ - 101

26. MUTOMBO DP., KRUBWA Y., KALUNDA M.

Infections postopératoires précoces en chirurgie ostéoarticulaire à Kinshasa : étude préliminaire de facteurs pathogéniques à propos de 189 interventions.

Médecine d'Afrique Noire 1993 ; 40(7)

\section{MINISTERE DE LA SANTE ET DE LA PROTECTION} SOCIALE

Programme National de Lutte contre les Infections Nosocomiales 2005 - 2008 ; page 2

28. Comite d'Infectiologie de I'Association Française d'Urologie (CIAFU)

GIROU E., BRUN-BUISSON C. Incidence et facteurs de risques des infections nosocomiales en urologie Enquête CIAFU (1998-1999) : résultats préliminaires ; page : 18

29. OTHEPA MO., RIU T.

Etude d'infection postopératoire dans un Hôpital rural du Zaïre

Congo médical 1994 ; $8: 424$ - 426

30. Réseau d'Alerte, d'Investigation et de Surveillance des Infections Nosocomiales (RAISIN)

Enquête nationale de prévalence des Infections Nosocomiales 2001 ; page : 26

31. CHADLI M., RTABI N., ALKANDRY S., KOEK JP., ACHOUR A., BUISSON Y., et all. Incidence de l'infection du site opératoire : étude prospective à l'Hôpital militaire d'instruction Mohamed - V de Rabat, Maroc

Disponible sur le site

http://www.sciencedirect.com/science?

Consulté le11/10/2008 vers $18 \mathrm{H} 40$

32. Epidémiologie de l'infection nosocomiale en maternité. Quelles sont les mesures dont l'efficacité est établie ? In Guide pour la surveillance et la prévention des Infections Nosocomiales en maternité Version 1 - Mai
1998 Version 2 - Juin 2003 Page 10 Disponible sur : WWW.sfhh.net

Liste des tableaux et figure

Tableau II : Les caractéristiques liées à l'infection de la plaie opératoire chez les opérés à l'hôpital Zone de Ouidah de 2006 à 2007

\begin{tabular}{|c|c|c|}
\hline Variables & $\begin{array}{l}\text { Effectif } \\
n=119\end{array}$ & $\%$ \\
\hline \multicolumn{3}{|l|}{$\begin{array}{l}\text { Infection de la plaie } \\
\text { opératoire }\end{array}$} \\
\hline Oui & 119 & 22,8 \\
\hline Non & 402 & 77,2 \\
\hline \multicolumn{3}{|l|}{$\begin{array}{l}\text { Structures intéressées } \\
\text { par l'infection }\end{array}$} \\
\hline $\begin{array}{l}\text { Peau et tissu sous cutané } \\
\text { sus aponévrotique }\end{array}$ & 39 & 32,8 \\
\hline Aponévroses et muscles & 31 & 26,1 \\
\hline $\begin{array}{l}\text { Organes et cavités } \\
\text { profondes }\end{array}$ & 49 & 41,2 \\
\hline \multicolumn{3}{|l|}{$\begin{array}{l}\text { Liquide/sérosité dans la } \\
\text { plaie }\end{array}$} \\
\hline Oui & 117 & 98,3 \\
\hline Non & 2 & 1,7 \\
\hline \multicolumn{3}{|l|}{$\begin{array}{l}\text { Signes locaux } \\
\text { d'infection }\end{array}$} \\
\hline Douleur & 65 & 54,6 \\
\hline Tuméfaction & 5 & 4,2 \\
\hline Rougeur & 4 & 3,4 \\
\hline Chaleur & 45 & 37,8 \\
\hline \multicolumn{3}{|l|}{$\begin{array}{l}\text { Déhiscence spontanée } \\
\text { de la plaie }\end{array}$} \\
\hline Oui & 30 & 25,2 \\
\hline Non & 89 & 74,8 \\
\hline \multicolumn{3}{|l|}{$\begin{array}{l}\text { Ré intervention sur la } \\
\text { plaie }\end{array}$} \\
\hline Oui & 19 & 16,0 \\
\hline Non & 100 & 84,0 \\
\hline
\end{tabular}


Tableau I : Les caractéristiques socio démographiques et les données cliniques des opérés à l'hôpital Zone de Ouidah de 2006 à $2007(n=521)$

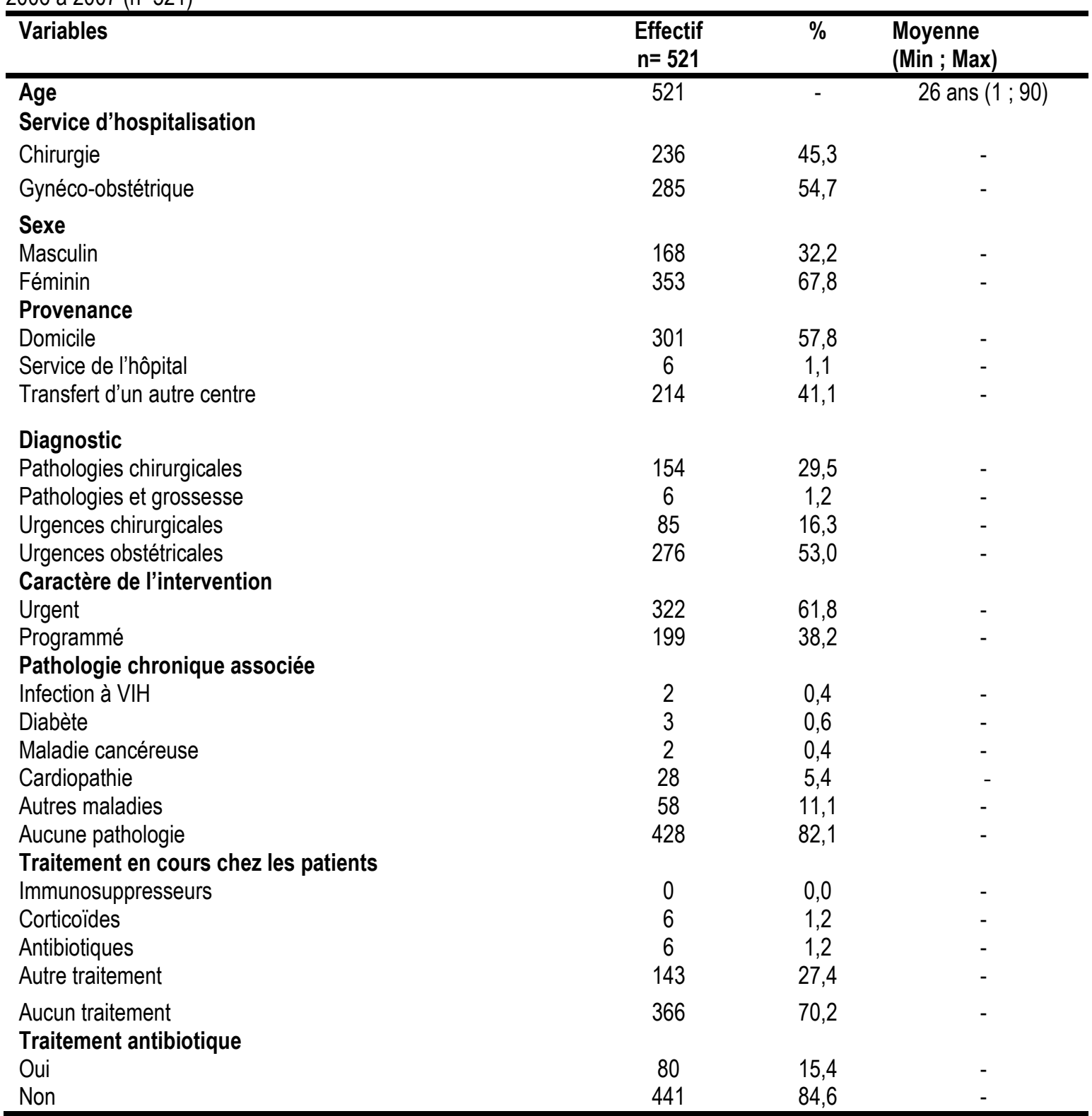


Tableau III : Les caractéristiques liées à l'acte chirurgical chez les opérés à l'hôpital Zone de Ouidah de 2006 à 2007(n=521).

\begin{tabular}{|c|c|c|}
\hline Variables & $\begin{array}{l}\text { Effectif } \\
n=521\end{array}$ & $\%$ \\
\hline \multicolumn{3}{|c|}{ Duré du séjour pré opératoire } \\
\hline 1 jour & 122 & 23,4 \\
\hline$>1$ jour & 75 & 14,4 \\
\hline$<1$ jour & 324 & 62,2 \\
\hline \multicolumn{3}{|c|}{ Rasage du champ opératoire } \\
\hline Oui & 451 & 86,6 \\
\hline Non & 70 & 13,4 \\
\hline \multicolumn{3}{|c|}{ Antibioprophylaxie } \\
\hline Oui & 509 & 97,7 \\
\hline Non & 12 & 2,3 \\
\hline \multicolumn{3}{|c|}{ Durée de l'intervention } \\
\hline 1 heure & 60 & 11,5 \\
\hline $1-2$ heures & 140 & 26,9 \\
\hline$>2$ heures & 321 & 61,6 \\
\hline \multicolumn{3}{|c|}{ Mise en place de drain } \\
\hline Oui & 82 & 15,7 \\
\hline Non & 439 & 84,3 \\
\hline \multicolumn{3}{|c|}{ Délai de retrait du drain } \\
\hline$\leq 4$ jours & 58 & 70,7 \\
\hline$>4$ jours & 24 & 29,3 \\
\hline \multicolumn{3}{|c|}{ Durée du séjour postopératoire } \\
\hline 1 semaine & 235 & 45,1 \\
\hline 2 semaines & 180 & 34,5 \\
\hline 3 semaines & 43 & 8,3 \\
\hline 4 semaines & 13 & 2,5 \\
\hline 5 semaines & 50 & 9,6 \\
\hline \multicolumn{3}{|c|}{ Durée du suivi postopératoire } \\
\hline$<30$ jours & 2 & 0,4 \\
\hline 30 jours & 515 & 98,8 \\
\hline$>30$ jours & 4 & 0,8 \\
\hline \multicolumn{3}{|c|}{ Rythme du pansement } \\
\hline Chaque jour & 90 & 17,3 \\
\hline Un jour sur 2 & 6 & 1,2 \\
\hline Un jour sur 4 & 425 & 81,6 \\
\hline Autres & 0 & 0,0 \\
\hline \multicolumn{3}{|c|}{ Mise en place d'implant / prothèse } \\
\hline Oui & 2 & 0,4 \\
\hline Non & 519 & 99,6 \\
\hline \multicolumn{3}{|c|}{ Mode de sortie des patients opérés } \\
\hline Guérison & 498 & 95,6 \\
\hline Evasion & 7 & 1,3 \\
\hline Transfert & 10 & 1,9 \\
\hline Décès & 6 & 1,2 \\
\hline
\end{tabular}


Tableau IV : étude de la relation entre l'infection de la plaie opératoire et les caractéristiques sociodémographiques des opérés à l'hôpital Zone de Ouidah de 2006 à 2007 (n=521) : analyse uni variée

\begin{tabular}{|c|c|c|c|c|c|}
\hline \multirow[t]{2}{*}{ Variables } & \multicolumn{2}{|c|}{$\begin{array}{c}\text { Infection de la plaie } \\
\text { opératoire }\end{array}$} & \multirow[t]{2}{*}{ OR } & \multirow[t]{2}{*}{ IC à $95 \%$} & \multirow[t]{2}{*}{ p-value } \\
\hline & $\begin{array}{c}\text { Oui } \\
119\end{array}$ & $\begin{array}{l}\text { Non } \\
402\end{array}$ & & & \\
\hline Age (années) & & & & & 0,0029 \\
\hline $1-30$ & 69 & 267 & 1 & - & - \\
\hline $30-60$ & 36 & 120 & 1,16 & $0,74-1,83$ & - \\
\hline$>60$ & 14 & 15 & 3,61 & $1,66-7,84$ & - \\
\hline Sexe & & & & & 0,032 \\
\hline Masculin & 48 & 120 & 1 & - & - \\
\hline Féminin & 71 & 282 & 0,63 & $0,41-0,96$ & - \\
\hline Service d'hospitalisation & & & & & 0,0007 \\
\hline Chirurgie & 70 & 166 & 1 & - & - \\
\hline Gynéco-obstétrique & 49 & 236 & 0,49 & $0,33-0,75$ & - \\
\hline
\end{tabular}

Tableau V : étude de la relation entre l'infection de la plaie opératoire et les données cliniques des opérés à l'hôpital Zone de Ouidah de 2006 à 2007(n=521) : analyse uni variée

\begin{tabular}{|c|c|c|c|c|c|}
\hline \multirow[t]{2}{*}{ Variables } & \multicolumn{2}{|c|}{$\begin{array}{c}\text { Infection de la plaie } \\
\text { opératoire }\end{array}$} & \multirow[t]{2}{*}{ OR } & \multirow[t]{2}{*}{ IC à $95 \%$} & \multirow[t]{2}{*}{$p$-value } \\
\hline & $\begin{array}{l}\text { Oui } \\
119\end{array}$ & $\begin{array}{l}\text { Non } \\
402 \\
\end{array}$ & & & \\
\hline Traitement antibiotique & & & & & $10^{-4}$ \\
\hline Oui & 35 & 45 & 1 & - & - \\
\hline $\begin{array}{l}\text { Non } \\
\text { Caractère de } \\
\text { l'intervention }\end{array}$ & 84 & 357 & 0,30 & $0,18-0,50$ & $\begin{array}{c}- \\
0,74\end{array}$ \\
\hline Programmé & 47 & 152 & 1 & - & - \\
\hline Urgent & 72 & 250 & 0,93 & $0,61-1,42$ & - \\
\hline Pathologies associées & & & & & 0,95 \\
\hline Non & 98 & 330 & 1 & - & - \\
\hline Oui & 21 & 72 & 0,98 & $0,57-1,68$ & - \\
\hline Traitement associé & & & & & $<19.10^{-4}$ \\
\hline Non & 70 & 296 & 1 & - & - \\
\hline Oui & 49 & 106 & 1,95 & $1,28-2,99$ & - \\
\hline
\end{tabular}


Tableau VI : Modèle initial

\begin{tabular}{|c|c|c|c|c|c|}
\hline \multirow{3}{*}{ Variables } & \multicolumn{2}{|c|}{$\begin{array}{l}\text { Infection de la plaie opératoire } \\
n=521\end{array}$} & \multirow{3}{*}{$\mathrm{OR}_{\mathrm{a}}$} & \multirow{3}{*}{ IC à 95\% } & \multirow{3}{*}{ p-value } \\
\hline & Oui & Non & & & \\
\hline & 119 & 402 & & & \\
\hline Age (années) & & & & & $>0,10$ \\
\hline $1-30$ & 69 & 267 & 1 & - & \\
\hline $30-60$ & 36 & 120 & 1,68 & {$[0,94-2,98]$} & - \\
\hline$>60$ & 14 & 15 & 2,21 & {$[0,67-7,31]$} & - \\
\hline Antibioprophylaxie & & & & & 0,96 \\
\hline Oui & 35 & 45 & 1 & - & \\
\hline Non & 84 & 357 & 0 & {$[0,00-1,0]$} & \\
\hline Classes d'antibiotiques & & & & & 0,06 \\
\hline$\beta$ lactamines & 72 & 316 & 1 & - & - \\
\hline Autres classes & 47 & 82 & 1,81 & {$[0,98-3,36]$} & - \\
\hline Mise en place de drain & & & & & 0,77 \\
\hline Non & 68 & 371 & 1 & - & - \\
\hline Oui & 51 & 31 & 0,82 & {$[0,21-3,19]$} & - \\
\hline Durée d'hospitalisation & & & & & $\leq 0,01$ \\
\hline 1 semaine & 14 & 221 & 1 & - & - \\
\hline$>1$ semaine & 69 & 181 & 11,97 & {$[5,69-25,20]$} & - \\
\hline Durée de l'intervention & & & & & $\underline{0,02}$ \\
\hline 1 heure & 3 & 57 & 1 & - & - \\
\hline$>1$ heure & 116 & 345 & 6,98 & {$[1,32-37,07]$} & - \\
\hline Rythme du pansement & & & & & $\leq 0,01$ \\
\hline Un jour sur deux & 56 & 40 & 1 & - & - \\
\hline Un jour sur quatre & 63 & 362 & 0,11 & {$[0,03-0,46]$} & - \\
\hline Rasage du champ opératoire & & & & & 0,14 \\
\hline Non & 23 & 47 & 1 & - & - \\
\hline Oui & 96 & 335 & 1,97 & {$[0,80-4,86]$} & - \\
\hline Service d'hospitalisation & & & & & 0,85 \\
\hline Chirurgie & 70 & 166 & 1 & - & - \\
\hline Gynéco-obstétrique & 49 & 236 & 0,89 & {$[0,27-2,93]$} & - \\
\hline Sexe & & & & & 0,62 \\
\hline Masculin & 48 & 120 & 1 & - & - \\
\hline Féminin & 71 & 282 & 1,26 & {$[0,51-3,15]$} & - \\
\hline Traitement associé & & & & & 0,74 \\
\hline Non & 70 & 296 & 1 & - & - \\
\hline Oui & 49 & 106 & 0,9 & {$[0,47-1,70]$} & - \\
\hline Types de plaies & & & & & 0,51 \\
\hline Plaies propres & 47 & 268 & 1 & - & - \\
\hline Plaies contaminées et sales/infectées & 72 & 134 & 1,29 & {$[0,60-2,77]$} & - \\
\hline
\end{tabular}


Tableau VII : Analyse multi variée en régression logistique des facteurs influençant la survenue de l'infection des plaies opératoires chez les opérés à l'hôpital Zone de Ouidah de 2006 à 2007 (n=521) : modèle Final

\begin{tabular}{|c|c|c|c|c|c|}
\hline \multirow[t]{2}{*}{ Facteurs } & \multicolumn{2}{|c|}{$\begin{array}{c}\text { Infection de la plaie } \\
\text { opératoire } n=521\end{array}$} & \multirow[t]{2}{*}{ ORa } & \multirow[t]{2}{*}{ IC à 95\% } & \multirow[t]{2}{*}{ p-value } \\
\hline & $\begin{array}{l}\text { Oui } \\
119\end{array}$ & $\begin{array}{l}\text { Non } \\
402\end{array}$ & & & \\
\hline Antibioprophylaxie & & & & & 0,96 \\
\hline Oui & 35 & 45 & 1 & - & \\
\hline Non & 84 & 357 & 0,00 & {$[0,00-1,0]$} & \\
\hline Classes d'antibiotiques & & & & & 0,03 \\
\hline$\beta$ lactamines & 72 & 316 & 1 & & \\
\hline Autres classes & 47 & 82 & 1,92 & {$[1,06-3,49]$} & \\
\hline Durée d'hospitalisation & & & & & $<0,01$ \\
\hline 1 semaine & 14 & 221 & 1 & - & \\
\hline$>=2$ semaines & 69 & 37 & 10,78 & {$[5,33-21,79]$} & \\
\hline Durée de l'intervention & & & & & 0,02 \\
\hline 1 heure & 3 & 57 & 1 & - & \\
\hline$>=2$ heures & 74 & 247 & 6,80 & {$[1,43-32,46]$} & \\
\hline Rythme du pansement & & & & & $<0,01$ \\
\hline Un jour sur deux & 56 & 40 & 1 & - & \\
\hline Un jour sur quatre & 63 & 362 & 0,12 & {$[0,06-0,24]$} & \\
\hline Rasage du champ opératoire & & & & & 0,16 \\
\hline Non & 23 & 47 & 1 & - & \\
\hline Oui & 96 & 335 & 1,86 & {$[0,78-4,41]$} & \\
\hline
\end{tabular}

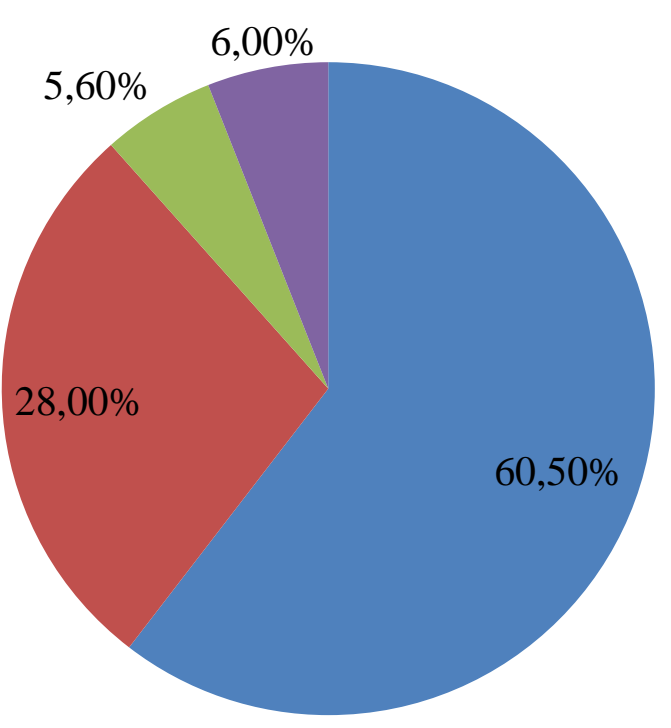

Plaie propre

Plaie propre contaminée

- Plaie contaminée

- Plaie sale / infectée

Figure 1 : Répartition des opérés selon les types de plaie à l'hôpital de Zone de Ouidah de 2006 à 2007. 